\title{
Janus kinase inhibitors: jackpot or potluck?
}

\author{
Keechilat Pavithran, Shripad Brahmanand Pande \\ Department of Medical Oncology and Hematology, Amrita Institute of Medical Sciences, Kochi, India
}

\begin{abstract}
The reports of a unique mutation in the Janus kinase-2 gene (JAK2) in polycythemia vera by several independent groups in 2005 quickly spurred the development of the Janus kinase inhibitors. In one of the great victories of translational research in recent times, the first smallmolecule Janus kinase inhibitor ruxolitinib entered a phase I trial in 2007. With the approval of ruxolitinib by the US Federal Drug Administration in November 2011 for high-risk and intermediate-2 risk myelofibrosis, a change in paradigm has occurred in the management of a subset of myeloproliferative neoplasms (MPN): primary myelofibrosis, post-polycythemia vera myelofibrosis, and post-essential thrombocythemia myelofibrosis. Whereas the current evidence for ruxolitinib only covers high-risk and intermediate-2 risk myelofibrosis, inhibitors with greater potency are likely to offer better disease control and survival advantage in patients belonging to these categories, and possibly to the low-risk and intermediate-1 risk categories of MPN as well. But use of the Janus kinase inhibitors also probably has certain disadvantages, such as toxicity, resistance, withdrawal phenomenon, non-reversal of histology, and an implausible goal of disease clone eradication, some of which could offset the gains. In spite of this, Janus kinase inhibitors are here to stay, and for use in more than just myeloproliferative neoplasms.
\end{abstract}

\section{Introduction}

The Philadelphia-negative myloproliferative neoplasms (MPN),

Correspondence: Keechilat Pavithran, Department of Medical Oncology and Hematology, Amrita Institute of Medical Sciences, Amrita Lane, Ponekkara, Edappally, Kochi - 682041, India.

Tel. +91.484.6681234 (Extension 3004).

E-mail: pavithrank@aims.amrita.edu

Key words: myeloproliferative neoplasms, myelofibrosis, polycythemia vera, essential thrombocythemia, janus kinase, ruxolitinib.

Received for publication: 18 March 2012.

Revision received: 24 May 2012.

Accepted for publication: 28 May 2012.

This work is licensed under a Creative Commons Attribution NonCommercial 3.0 License (CC BY-NC 3.0).

CC Copyright K. Pavithran and S.B. Pande, 2012

Licensee PAGEPress, Italy

Oncology Reviews 2012; 6:e13

doi:10.4081/oncol.2012.e13 polycythemia vera (PV), essential thrombocythemia (ET) and primary myelofibrosis (PMF), are characterized by a long and progressive course. The median survival of PMF is less than five years ${ }^{1}$ whereas, with the best available therapy, ET patients have close to normal survival $^{2}$ and the median survival of patients with PV is 19 years. ${ }^{3}$ Two inter-related processes are responsible for the symptoms in PV, ET and PMF, and myelofibrosis (MF) primary, post-ET or post-PV: i) clonal proliferation; and ii) a reactive inflammatory state. Since the reports of the discovery of the Janus kinase-2 gene (JAK2) ${ }^{V 617 F}$ mutation in PV in $2005,^{4-8}$ the last seven years have witnessed several advances in the understanding of these disorders. The observation that the $J A K 2^{V 617 F}$ mutation is found in $95 \% \mathrm{PV}$ and more than $50 \%$ in ET and MF quickly prompted the development of JAK2 protein inhibitors, which marks one of the great advances in translational research of recent times.

\section{Normal JAK2-mediated signaling}

The Janus kinase family of non-receptor tyrosine kinases includes four proteins: JAK1, JAK2, JAK3 and Tyk2. Growth factors such as erythropoietin, thrombopoietin and granulocyte colony stimulating factor bind to their cognate receptors at the cell membrane; JAK2 associates with these receptors, like an adapter, for the downstream transduction of signals to the nucleus through the JAK-STAT pathway. The binding of cytokine ligand to the cytokine receptors results in activation of the receptors and consequent autophosphorylation of JAK2. The phosphorylation of tyrosines serves as docking sites for the recruitment and assembly of downstream signaling proteins. This in turn results in activation of specific cascades involving STAT, MAPK, ERK and P-I-3 KinaseAKT. Negative feedback mechanisms involving silencer of cytokine signaling (SOCS), CBL, LNK and other proteins attenuate the signaling. ${ }^{9}$

\section{$J A K 2^{\mathrm{V} 617 \mathrm{~F}}$ mutation in myloproliferative neoplasms}

In 2005, four groups reported a specific mutation of the JAK2 gene, i.e. JAK2 $2^{V 617 F}$ in PV, ET and PMF. The mutation is observed with a frequency of over $95 \%$ in PV, 32-57\% in ET and $35-50 \%$ in PMF.,7 The $J A K 2^{V 617 F}$ results from a guanine-to-thymidine transversion at nucleotide 1849 on exon 14 of JAK2 which translates into the substitution of valine by phenylalanine at position 617 in the pseudokinase domain of the JAK2 protein.-8 The gain-of-function mutation leads to a constitutive activation of JAK2 $2^{\mathrm{V} 617 \mathrm{~F}}$ and an uncontrolled activation of the downstream pathway called JAK-STAT pathway, in addition to some other pleotropic effects of JAK2 ${ }^{\mathrm{V} 617 \mathrm{~F}}$.

Of the seven domains of the JAK2 protein, the mutation hits the pseudokinase $\mathrm{JH} 2$ domain. JH2 has an auto-inhibitory effect on JH1 which is the kinase domain of JAK2. The substitution of phenylalanine 
at position 617 in $\mathrm{JH} 2$ domain results in a pi-stacking of phenylalanine residues and changed physical characteristics of JAK2. ${ }^{10}$ This relieves the kinase domain of the inhibition from $\mathrm{JH} 2$, which is rendered perpetually switched-on. JAK2 ${ }^{\mathrm{V} 617 \mathrm{~F}}$ hyperphosphorylates attenuating SOCS3 protein and exploits it in ensuring the intense signal transduction duration is prolonged. ${ }^{11}$ When $J A K 2^{V 617 F}$ is expressed in hematopoietic cells, several signaling pathways, including STAT3, STAT5, MAPK, ERK and PI3K-AKT, are overactivated. The net effect is proliferation, survival and differentiation in hematopoietic cells leading to the MPN phenotype.

In addition to the two gains referred to above, there is at least one additional epigenetic effect conferred by the JAK2 ${ }^{V 617 F}$ mutation: JAK2 ${ }^{\mathrm{V} 617 \mathrm{~F}}$ translocates to the nucleus and phosphorylates PRMT5 incapacitating it from methylating histone $\mathrm{H} 2 \mathrm{~A}$ and $\mathrm{H} 4$ (on specific arginine residues). Abrogation of PRMT5 may also contribute to the MPN phenotype. ${ }^{9}$

\section{Exon 12 and other mutations}

Recurring mutations on $J A K 2$ other than in exon 14 have been observed in exon 12. Exon 12 mutations are observed roughly in onethird of patients with $J A K 2^{V 617 F}$-negative PV; the overall incidence in PV is $3 \%$. The frequency of other mutations such as $M P L, L N K, C B L, I D H 1$, IDH2, TET2, EZH2, DNMT3A, ASXL1, SF3B1, IKZF1, TP53, CUX1 and others $^{12,13}$ is less than $20 \%$ and often below $10 \%{ }^{13}$ (Table 1).

\section{Targeting the Janus kinase in myelofibrosis to bridge the gap between the need and the availability}

All MPNs, irrespective of the frequency of $J A K 2^{V 617 F}$ mutation, have the same basic underlying pathophysiology: clonal myeloproliferation and hyperreactiveness to cytokines. ${ }^{9}$ ET, PV, PMF and post-ET/PV MF, all harbor the same somatic mutations albeit with variable frequencies. Since ET and PV have a very long natural history with median survival of decades, and are amenable to control with the best available therapy (Table 2), there does not seem to be an urgent need of a drug for these diseases. However, PMF and post-PV/ET MF are characterized by shorter median survival and a greater severity of symptoms, including constitutional symptoms. There was, therefore, an urgent need for a new therapy in these patients, and in particular in the worse subset. Hence, though the activity of Janus kinase inhibitors in PV and ET has been and is being tested, for the moment, the main focus of the Janus kinase inhibitor trials is on MF.

The Janus kinase inhibitors are small-molecule ATP inhibitors. They exert their effect by competing with the ATP-binding site on the kinase. $^{14}$ The first among these, ruxolitinib (Jakafi, Incyte Corp.,

Table 1. Mutations associated with myloproliferative neoplasms, and likely targeted therapy.

\begin{tabular}{lcc} 
Pathway & Mutations & Targeted therapy \\
JAK/STAT & $J A K 2^{V 617 F}$, JAK2 exon & Ruxolitinib and others \\
PI3K-AKT-Mtor & 12, MPL, CBL, LNK & Everolimus \\
Epigenetic & TET2, ASXL1, EZH2 & Givinostat, \\
& & Panobinostat, vorinostat \\
\hline Oncogenic & $I D H$ & - \\
\hline
\end{tabular}

Wilmingtom, DE, USA), which was introduced in trials in 2007, was approved by the US Federal Drug Administration for use in high-risk and intermedicate- 2 risk MF in November 2011 on the basis of phase III data. ${ }^{15}$ There are a number of Janus kinase inhibitors currently in clinical trials at various stages of development (Table 3).

Janus kinase inhibitors differ from one another with respect to: i) their ability to inhibit one or the other Janus kinase to different degrees; ii) their specificity for mutated rather than wild-type kinase; iii) hematologic and non-hematologic toxicities; iv) off-target activity; v) potential for reducing the $J A K 2^{V 617 F}$ allele burden; and vi) activity against other kinases such as FLT.

\section{Ruxolitinib (JAKAFI)}

\section{Pre-clinical evidence of ruxolitinib activity in myloproliferative neoplasms}

Ruxolitinib has potent inhibitory activity against JAK 1 and 2, moderate activity against TYK2 and negligible activity against JAK 3 . In $\mathrm{Ba} / \mathrm{F} 3$ cells expressing $J A K 2^{V 617 F}$, ruxolitinib induced dramatic inhibi-

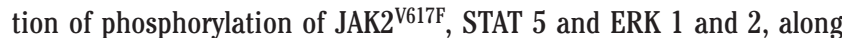
with reduced cellular proliferation and induction of apoptosis. Ruxolitinib potently inhibited the proliferation of ex vivo expanded erythroid progenitors obtained from patients with $J A K 2^{V 617 F}$-positive PV. In a murine model of $J A K 2^{V 617 F}$-driven malignancy, ruxolitinib reversed the disease with normalization of histology and reduction of spleen, and prolonged survival. Circulating levels of pro-inflammatory cytokines, such as IL-6 and TNF-alpha, believed to be responsible for the constitutional symptoms in PMF, were dramatically reduced in this model..$^{16}$

Table 2. Traditional therapies for myloproliferative neoplasms.

\begin{tabular}{|c|c|c|}
\hline & Objective & Options \\
\hline PV & $\begin{array}{l}\text { To avoid hemorrhagic and } \\
\text { thrombotic complications }\end{array}$ & $\begin{array}{c}\text { Low risk: phlebotomy } \\
\text { frontline (Target hct }<45 \text { in } \\
\text { men and }<42 \text { in women) } \\
\text { High risk: phlebotomy+ } \\
\text { Aspirin } \\
\text { HU in patients exhibiting } \\
\text { poor response to aspirin } \\
\text { HU+Aspirin }\end{array}$ \\
\hline ET & $\begin{array}{l}\text { To avoid hemorrhagic and } \\
\text { thrombotic complications }\end{array}$ & $\begin{array}{l}\text { Low risk: no therapy } \\
\text { Intermediate risk: Aspirin } \\
\text { High risk: HU standard, } \\
\text { Aspirin }\end{array}$ \\
\hline \multirow[t]{3}{*}{ PMF } & Improvement of cytopenias & $\begin{array}{l}\text { Corticosteroids, danazol, } \\
\text { erythropoietic stimulating }\end{array}$ \\
\hline & Reduction in splenomegaly & $\begin{array}{l}\text { HU, irradiation or } \\
\text { splenectomy } \\
\text { Lenalidomide-Prednisolone } \\
\text { combination }\end{array}$ \\
\hline & Curative intent & $\begin{array}{l}\text { Allogeneic stem-cell } \\
\text { transplant }\end{array}$ \\
\hline
\end{tabular}

PV, polycythemia vera; ET, essential thrombocythemia; PMF, primary myelofibrosis; HU, hydroxyurea: hct, hematocrit. 


\section{Clinical evidence of ruxolitinib efficacy in myelofibrosis: phase I/II}

Safety and efficacy of ruxolitinib was tested in a phase I/I trial of 153 patients of PMF, post-ET or post-PV myelofibrosis. ${ }^{17}$ The dose-limiting toxicity was grade 4 thrombocytopenia. After 28 days of therapy, there were dramatic reductions in fibrogenic, angiogenic and pro-inflammatory growth factors independent of JAK2 mutational status. ${ }^{17}$ Mean leukocyte count after three months of treatment (15 or $25 \mathrm{mg}$ twice-daily) decreased from $29.8 \times 10^{9} / \mathrm{L}$ to $16.0 \times 10^{9} / \mathrm{L}$, and 7 (44\%) of 16 patients with baseline thrombocytosis normalized their platelet count. After a median follow up of approximately 15 months, the anemia, spleen ( $>50 \%$ reduction on palpation) and constitutional-symptoms (scored by Myelofibrosis Symptoms Assessment Form) response rates were 14\%, 44\% and more than $50 \%$, respectively. ${ }^{17}$ Ruxolitinib was found to have little effect on $J A K 2^{\text {V617F }}$ allele burden or bone marrow fibrosis. The improvements with ruxolitinib were independent of mutational status or origin of myelofibrosis (primary or post-PV/ET). Non-hematologic toxicity occurred in less than $10 \%$ of patients and was commonly grade 1 or 2 . Thrombocytopenia (grade 3,17\%; grade 4,3\%) and treatment-emergent anemias (23\%) were the most common adverse effects. ${ }^{18}$

\section{Clinical evidence of ruxolitinib efficacy in polycythemia vera and essential thrombocythemia: phase II}

Ruxolitinib activity was also tested in a phase II study of PV ( $\mathrm{n}=34)$ and ET $(n=39)$ patients refractory or intolerant to hydroxyurea (HU). In patients with $\mathrm{PV}$, significant reductions in hematocrit, leukocyte count and platelet count were observed at six months, with a complete response rate of $45 \%$ and partial response rate of $52 \%$ according to European Leukemia Net response criteria. ${ }^{19,20}$ In patients with ET, up to $75 \%$ patients experienced a reduction in pruritus, bone pain, night sweats, and an at least $50 \%$ reduction in peripheral neuropathy scores. The complete and partial response rates were $13 \%$ and $77 \%$, respectively. Toxicity in this study was less than grade 4 : grade 2 anemia in $12 \%$ $\mathrm{PV}$ and $18 \% \mathrm{ET}$, and grade 3 leukopenia in 9\% PV and 5\% ET. ${ }^{19,20}$

\section{Clinical evidence of ruxolitinib superiority in high- and intermedicate-2 risk myelofibrosis: phase III (COMFORT I)}

Ruxolitinib was tested in two phase III trials: COMFORT I and II (controlled myelofibrosis study with oral JAK inhibitor treatment). COMFORT I compared ruxolitinib $(n=155)$ with placebo $(n=154)$ in International Prognostic Scoring System ${ }^{21}$ high-risk or intermediate-2 risk $\mathrm{MF}^{22}$ Continuous oral administration of ruxolitinib was given at doses of $15 \mathrm{mg}$ twice a day (platelets $100-200 \times 10^{9} / \mathrm{L}$ ) or $20 \mathrm{mg}$ twice a day (platelets $>200 \times 10^{9} / \mathrm{L}$ ).

The primary end point was regression of spleen volume by $35 \%$ or more with computed tomography or magnetic resonance imaging (MRI) at the end of 24 weeks. The secondary end point was symptom improvement. In addition, there were some exploratory end points. In the ruxolitinib arm, approximately $45 \%$ had PMF, $32 \%$ had post-PV MF and $23 \%$ had post-ET MF, whereas the respective percentages in the control arm were $55 \%, 32 \%$ and $14 \%$. The main patient distribution data of the two COMFORT trials (COMFORT I and II) have been summarized in Table 4.

At 24 weeks, spleen response (primary end point) was $41.9 \%$ with ruxolitinib versus $0.7 \%$ on placebo. Almost all patients on ruxolitinib had some spleen response and the majority of patients receiving placebo had growth in spleen size (Figure 1). In addition, $45.9 \%$ of patients on ruxolitinib versus $5.3 \%$ on placebo experienced a $50 \%$ or more improvement in constitutional symptoms. Most patients on ruxolitinib had improvement in symptoms and the majority of patients receiving placebo had a worsening of symptoms. The benefits were apparent among all subtypes of MF irrespective of JAK2 mutational status, reflecting data obtained from a phase II study. Ten deaths occurred in the ruxolitinib arm and 14 in the placebo arm. The authors reported a statistically significant survival benefit with ruxolitinib. ${ }^{22}$

Grade 3 and grade 4 anemia with ruxolitinib was $45.2 \%$ compared with $19.2 \%$ with placebo; and grade 3 and grade 4 thrombocytopenia

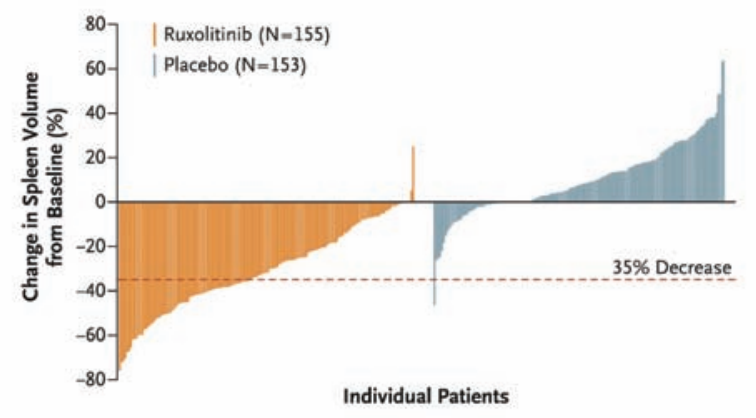

Figure 1. Waterfall diagram showing the percent change in spleen volume from baseline (with permission: COMFORT I, Verstovsek et al., New England Journal of Medicine 2012).

Table 3. The Janus kinase inhibitors: selectivity and phase of development.

\begin{tabular}{|c|c|c|c|c|}
\hline Compound & JAK2 vs. JAK3 & $\begin{array}{l}\text { ectivity against } \\
\text { JAK2 vs. JAK1 }\end{array}$ & JAK2 vs. TYK2 & $\begin{array}{c}\text { Stage of } \\
\text { developmen }\end{array}$ \\
\hline Ruxolitinib & 153 & 1.1 & 6.7 & Phase 3 \\
\hline TG101348 (SAR302503) & 332 & 35 & 135 & Phase 3 \\
\hline Lestaurtinib & 3 & N/A & N/A & Phase 2 \\
\hline XL019 & 125 & 65 & 170 & Halted \\
\hline CYT387 & 8.6 & 0.6 & N/A & Phase $1 / 2$ \\
\hline AZD1480 & 15 & 5 & N/A & Phase $1 / 2$ \\
\hline SB1518 & 24 & 58 & N/A & Phase $1 / 2$ \\
\hline LY2784544 & N/A & N/A & N/A & Phase 1/2 \\
\hline
\end{tabular}

JAK, Janus kinase; N/A, not applicable or not available. 
was reported in $12.9 \%$ patients with ruxolitinib and $1.3 \%$ with placebo. The most frequent grade 3 and 4 non-hematologic toxicities in the study arm were abdominal pain, fatigue and dyspnea; these were more frequent in the placebo arm.

There was no difference in rate of withdrawal due to adverse events between the ruxolitinib (11\%) the placebo (10.6\%) arms. There was no clear pattern of any syndrome following withdrawal of ruxolitinib.

\section{Clinical evidence of ruxolitinib superiority} in high- and intermediate-2 risk myelofibrosis: phase III COMFORT II

This European study comparing ruxolitinib with best available treatment (BAT) examined spleen volume at the end of 48 weeks. ${ }^{23}$ Patients eligible had high- or intermediate-2 risk disease. Ruxolitinib was administered at doses of $15 \mathrm{mg}$ twice a day (platelets $<200 \times 10^{9} / \mathrm{L}$ ) or 20 mg twice a day (platelets $>200 \times 10^{9} / \mathrm{L}$ ). The principal end point was reduction in spleen volume by $35 \%$ or more (spleen response). Secondary end points were duration of spleen response and improvement in symptom score.

Spleen response was $32 \%$ at 24 weeks and $28 \%$ at 48 weeks versus $0 \%$ for BAT. At the time of reporting of the trial, the median duration of response had not reached and $80 \%$ were still holding the spleen response at a median follow up of 12 months. All subgroups derived benefit from ruxolitinib. Spleen response was seen in both mutationpositive and negative patients, but some numerical differences emerged. In $J A K 2^{V 617 F}$-positive patients, the spleen response rate for ruxolitinib was $33 \%$ (vs $0 \%$ with BAT), whereas in $J A K 2^{V 617 F}$-negative patients the spleen response was $14 \%$ (vs $0 \%$ with BAT).

At week 48, patients receiving ruxolitinib had marked reductions in myelofibrosis-associated symptoms (anorexia, dyspnea, fatigue, insomnia and pain) whereas patients receiving BAT had worsening symptoms measured with EORTC QLQ-C30 scores (Figure 2). The authors did not suggest any survival benefit with ruxolitinib. Whether survival benefit will be demonstrable at a longer follow up remains to be seen.
The most frequent combined grade 3 and 4 non-hematologic toxicity was diarrhea (1\%). Combined grade 3 and 4 anemia was $42 \%$ with ruxolitinib and $31 \%$ with best available therapy. Grade 3 and 4 thrombocytopenia was seen in $8 \%$ with ruxolitinib versus $7 \%$ with BAT. ${ }^{23}$ Overall, serious adverse events were infrequent in the ruxolitinib arm and were more frequent in patients receiving BAT. The number of patients discontinuing the study medication due to all-grade adverse events was small (8\%) and did not differ greatly from the number discontinuing BAT (5\%)

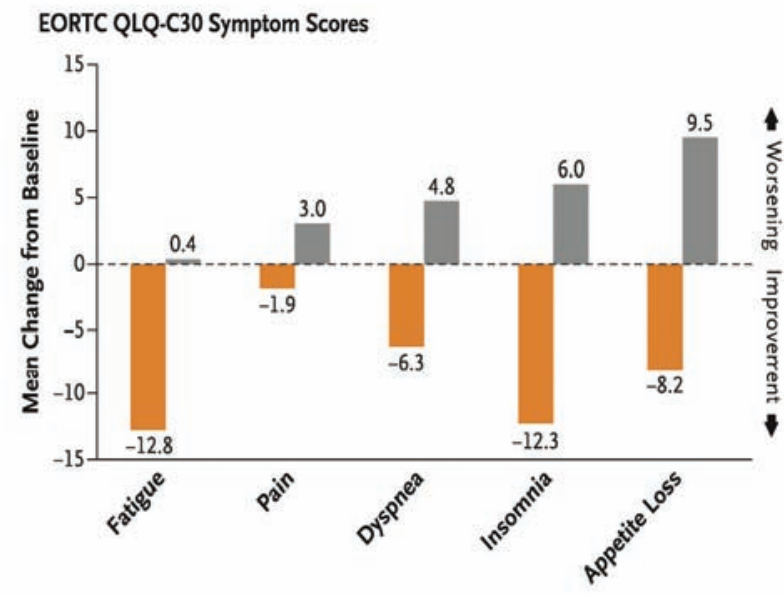

Figure 2. Mean changes in EORTC QLQ-C30 scores from baseline, showing improvement all symptoms with ruxolitinib and worsening of all symptoms with BAT. (with permission: COMFORT II, Harrison et al, New England Journal of Medicine 2012).

Table 4. Comparison of patient distribution and end points of COMFORT I and II.

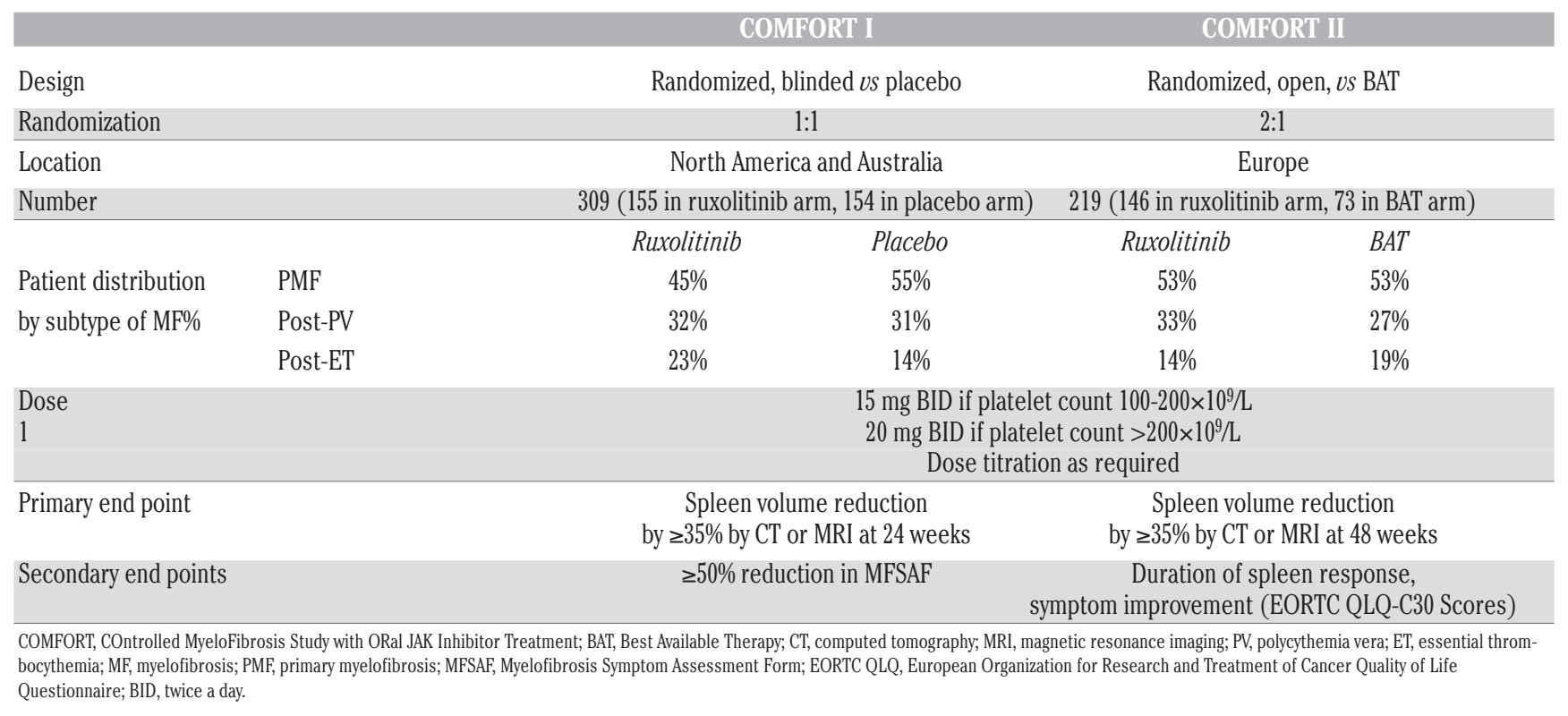




\section{Clinical evidence of ruxolitinib efficacy in polycythemia vera: phase III}

A phase III trial of ruxolitinib in patients with $\mathrm{HU}$ resistant or HU-intolerant PV is currently underway. ${ }^{15}$

\section{Long-term follow up of ruxolitinib a phase I/ II in myelofibrosis: the Mayo experience}

The follow up of Mayo Clinic patients of MF (one of the two participant centers, the other being MD Anderson Cancer Center) in a phase I/I ruxolitinib trial $(n=51)$ was updated in July 2011. The overall response rate was reported to be $29 \%$ for spleen, $21 \%$ for anemia and $63 \%$ for constitutional symptoms. Most frequent grade 2,3 and 4 toxicities were: thrombocytopenia (26\%) and anemia (33\%). Cumulative treatment discontinuation rate at one year was $51 \%$, at two years $72 \%$ and at three years $89 \%$. The most common causes for discontinuation were loss of treatment response and toxicity. A rapid rebound in symptoms following drug discontinuation, sometimes requiring hospitalization, was seen in almost all such cases. After comparison of the riskadjusted survival of the Mayo cohort of ruxolitinib-treated patients with 410 untreated patients, no survival advantage was seen. ${ }^{24}$

\section{Long-term follow up of ruxolitinib phase I/ II: the MD Anderson experience}

Of 107 patients in a phase I/II ruxolitinib trial at the MD Anderson Cancer Center, $54 \%$ were still continuing treatment at a median follow up of 32 months. After comparison with historic patients, authors concluded that there was a survival benefit with ruxolitinib. ${ }^{25}$

\section{Other Janus kinase inhibitors in trials}

There are many other JAK inhibitors which have undergone phase I/I trials in humans. One has entered phase III (SAR302503) while the development of another trial (XL019) was halted following druginduced neuropathy.

\section{SAR302503 (TG101348)}

This is the only Janus kinase inhibitor after ruxolitinib to have entered into a phase III trial in high-risk and intermediate-2 risk MF patients against placebo. At the time of writing, phase III interim data have still not been.

In a phase I/I experience, DLT was hyperamylasemia. Independent of $J A K 2$ mutational status, 6 cycles of treatment with SAR302503 yielded a $39 \%$ palpable spleen response (defined as $\geq 50 \%$ regression) and constitutional symptom response (defined as $\geq 50 \%$ reduction). Leukocytosis response (defined as normalization) was $72 \%$ and thrombocytosis response was $90 \%$. Common side effects were nausea and diarrhea (majority of patients), transfusion dependency (35\%), grade 3 or 4 thrombocytopenia (24\%), and asymptomatic increases in serum lipase (27\%) and transaminases (27\%). ${ }^{26}$ After 24 cycles of treatment, there was a statistically significant decrease in $J A K 2^{V 617 F}$ allele burden from a baseline of median $20 \%(\mathrm{n}=51$; range $3-100 \%)$ to median $9 \%$ $(\mathrm{n}=21$; range $0-100 \%){ }^{27}$

\section{CYT387}

In a phase I/I multi-center study CYT387, 163 patients with high- or intermediate- 2 risk MF had been enrolled at the time of the most recent study report. ${ }^{28}$ DLT included grade 3 hyperlipasemia and grade 3 headaches. For the initial 60 patients completing at least 3 cycles of treatment with CYT387, spleen, anemia and constitutional symptom response rates (by conventional criteria) ${ }^{29}$ were $45 \%, 50 \%$ and $50 \%$ or over, respectively, irrespective of $J A K 2^{V 617 F}$ mutational status. ${ }^{30}$ Interestingly, $58 \%$ of transfusion-dependent patients became transfusion-independent. ${ }^{30}$ The most common toxicity included transient lightheadedness and hypotension seen only with the first dose, and grade 3 or 4 thrombocytopenia seen in $16 \%$ of subjects. ${ }^{30}$ Grade 1 toxicities included peripheral sensory neuropathy whose incidence and natural history is currently being studied. A unique divergence in toxicity with respect to ruxolitinib and SAR302503 was the lack of treatment-related grade 3 or 4 anemia, which was less than $1 \%$. At the time of the most recent report, 25 (15\%) of the 163 study subjects had discontinued treatment.

\section{Lestaurtinib}

In a phase II study, ${ }^{31}$ lestaurtinib (CEP-701) at $80 \mathrm{mg}$ twice daily was given to $22 J A K 2$-mutated MF patients. Overall response by International Working Group for Myelofibrosis Research and Treatment (IWG-MRT) criteria $^{29}$ was $27 \%$. Among 8 transfusion-dependent patients, 2 (25\%) became transfusion-independent. Treatment had no impact on bone marrow histopathology, JAK2 ${ }^{V 617 F}$ allele burden or inflammatory cytokine levels. Side effects included diarrhea (73\%), nausea (50\%), and grade 3 or 4 anemia (14\%) and thrombocytopenia (23\%). After a median follow up of less than 1.5 years, 21 patients (91\%) discontinued therapy mostly because of lack of response and 6 (27\%) deaths were reported.

A phase I study of lestaurtinib with a new capsule formulation (as opposed to liquid formulation) to circumvent excess plasma protein binding is currently ongoing in patients with $J A K 2^{V 617 F}$-positive $\mathrm{MF}^{32}$

Results were disappointing in another phase II study of lestaurtinib in high-risk patients of $J A K 2^{V 617 F}$-positive PV $(\mathrm{n}=27)$ or ET $(\mathrm{n}=12)$. Five patients experienced a worsening of leukocytosis and thrombocytosis, and thrombosis. ${ }^{33}$

\section{SB1518 (pacritinib)}

The dose limiting-toxicity (DLT) during a phase I study of SB1518 diarrhea was an adverse event at a dose of $600 \mathrm{mg} /$ day. ${ }^{34}$ In the phase II part of the same study, MF patients not suitable for standard therapy were enrolled. Of 30 patients assessed by MRI, 17 (57\%) had a reduction in spleen-volume of $25 \%$ or more. There was a reduction in intensity of MF-related symptoms of $40-65 \%$ in patients treated for six months. The most common treatment-related toxicities were diarrhea (81\%; 6\% grade 3), nausea (41\%; all grade $1 / 2)$, vomiting (22\%; all grade $1 / 2$ ), and fatigue ( $9 \%$; all of grade $1 / 2$ ). At six months, 21 patients remain on therapy. There was no grade $3 / 4$ neutropenia or thrombocytopenia. SB1518 was tolerated equally well by patients with normal platelet counts and those with thrombocytopenia. ${ }^{35}$

In another phase II study in 34 patients of MF (PMF, post-ET/PV MF), spleen response rate was $44 \%$ by physical examination and $32 \%$ by MRI ( $\geq 35 \%$ reduction in splenic volume). Two patients met anemia response. Seventeen (50\%) had discontinued treatment, mainly because of adverse effects or disease progression. ${ }^{36}$

\section{LY2784544}

Among the 19 patients enrolled so far in a phase I study of LY2784544 in patients with PMF $(n=17)$, PV $(n=1)$ or ET (1), DLT included increases in uric acid and creatinine at a dose of $200-240 \mathrm{mg} /$ day related to tumor lysis syndrome (TLS). The patient with PV was reported to experience a $100 \%$ reduction in spleen size. In MF, 4 (22\%) patients have so far achieved spleen response. No responses have so far been observed in terms of $J A K 2^{\text {V617F }}$ allele burden reduction. Toxicity has mainly involved diarrhea (42\%), nausea (37\%), anemia (21\%) and transient increases in serum creatinine, uric acid, and potassium, some of which has been attributed to TLS. ${ }^{37}$ 


\section{XL019}

In a phase I study involving $21 \mathrm{MF}$ patients, 7 of 9 patients receiving XL019 at daily doses of $100 \mathrm{mg}$ or more experienced grade 1 or 2 peripheral or central neuropathy. At lower doses ( $\leq 50 \mathrm{mg} /$ day), used in the remaining patients, favorable effects were seen in JAK2 or MPL mutated patients but not in unmutated patients. However, because of neurological toxicities, further development of XL019 in trials has been halted. ${ }^{38}$

\section{BMS911543, NS-018 and AZD1480}

At present, no information is available on the studies undertaken with these drugs. ${ }^{15}$

\section{Janus kinase inhibitors: beyond myloproliferative neoplasms}

Inhibitors of one or the other type of Janus kinase are likely to be of use in hematologic malignancies and in benign disorders such as rheumatoid arthritis and other autoimmune diseases. ${ }^{14}$

\section{Targeting in myloproliferative neoplasms: beyond Janus kinase inhibitors}

Histone deacetylase inhibitors givinostat, panobinostat and vorinostat were documented to down-regulate the levels of phosphorylated $\mathrm{JAK} 2^{\mathrm{V} 617 \mathrm{~F}}$ in in vitro studies which led to the phase I/II studies with these agents in MPN.

\section{Givinostat}

In a phase IIA study of givinostat, a novel histone-deacetylase inhibitor, in patients with PV $(n=12)$, ET $(n=1)$ and MF $(n=16)$ bearing the $J A K 2^{V 617 F}$ mutation, givinostat was given orally for 24 weeks at a starting dose of $50 \mathrm{mg}$ twice daily. Ten patients discontinued treatment mainly due to disease, minor toxicity and withdrawal of consent. Among 13 PV/ET patients, one complete, 6 partial and 4 no responses were documented at study end while 2 patients went off-study prematurely. Three major responses were registered among $16 \mathrm{MF}$ patients. Pruritus disappeared in most patients, and reduction of splenomegaly was observed in $75 \%$ of PV/ET and 38\% of MF patients. Reverse transcription polymerase chain reaction identified a trend towards reduction of the JAK2 ${ }^{\mathrm{V} 617 \mathrm{~F}}$ allele burden. Givinostat was well tolerated and could induce hematologic response in most PV and some MF patients. ${ }^{39}$

\section{Panobinostat}

A phase I trial identified reversible thrombocytopenia as the DLT. ${ }^{40} \mathrm{~A}$ phase II trial by the same investigator group is ongoing, and had enrolled 14 patients at the time of the last reports. ${ }^{40}$

Interim data of another phase II trial was presented in abstract form in 2010. The authors reported that the majority of patients required dose reduction. However, specific adverse events, their frequency, and response rates were not reported in the 2010 abstract and no further reports of data fromthis study have been published. ${ }^{41}$

\section{Eeverolimus}

In addition to dysregulated JAK/STAT signaling, activation of the AKT/mTOR pathway occurs in MPN. In a phase I/II study with everolimus, an mTOR inhibitor, in 39 high- or intermediate-risk PMF or post-PV/ET myelofibrosis subjects, responses were evaluated in 30 patients of the phase II group..$^{42}$ No dose-limiting toxicity was observed in phase I up to $10 \mathrm{mg} / \mathrm{d}$. When this dose was used in phase II, grade 3 or more toxicities were infrequent; the most common toxicity was grade 1-2 stomatitis. Rapid and sustained reduction in splenomegaly of more than $50 \%$ and more than $30 \%$ occurred in $20 \%$ and $44 \%$ of subjects, respectively. A total of $69 \%$ and $80 \%$ patients experienced complete resolution of systemic symptoms and pruritus, respectively. Response in leukocytosis, anemia, and thrombocytosis occurred in 15$25 \%$. Clinical responses were not associated with reduced $J A K 2^{V 617 F}$ burden or cytokine levels. Response rate was $60 \%$ when European Network for Myelofibrosis criteria were used ( 8 major, 7 moderate, 3 minor responses) or $23 \%$ when IWG-MRT criteria were used (one partial response, 6 clinical improvements). These results provide proof-ofconcept that targeting mTOR pathway in myelofibrosis may be clinically relevant.

\section{Pomalidomide}

In a study of long-term follow-up data on patients from the Mayo Clinic who had participated in three consecutive clinical trials using single-agent pomalidomide for MF, the authors reported their observations on 82 patients with MF (primary and post-PV/ET) enrolled in phase I and II clinical trials. Forty-five (55\%), 24 (29\%), 7 (9\%), and 2 (2\%) patients remained on pomalidomide therapy for at least 6, 12, 24 and 36 months, respectively. The overall anemia response rate per IWGMRT criteria was 27\% (22 of 82). There was no report of spleen or symptom response in their abstract. The authors concluded that anemia response to pomalidomide therapy in myelofibrosis often occurs in the first six months of treatment and is more likely to occur in the presence of $J A K 2^{V 617 F}$ and absence of marked splenomegaly. Sensory peripheral neuropathy was reported to be likely with long-term therapy with pomalidomide. ${ }^{43}$

\section{Skepticism over Janus kinase inhibitors in myloproliferative neoplasms}

Though a clear palliative benefit with ruxolitinib has been established in MF by means of two phase III randomized controlled clinical trials, there are reasons to suspect shortcomings associated with the use of ruxolitinib, in particular, and all the Janus kinase inhibitors in general and, therefore, a few notes of caution are in order.

Currently, there is evidence of benefit from ruxolitinib only in highand intermediate- 2 risk MF patients. ${ }^{22,23}$ The low- and intermediate- 1 risk categories may not derive any benefit from ruxolitinib. The role of ruxolitinib needs to be verified in phase III trials for these patients; data for the high- and intermediate-2 risk patients cannot be overzealously extrapolated to low- and intermediate-1 risk categories to treat them with ruxolitinib.

Ruxolitinib therapy has toxicity and transfusion requirement. ${ }^{22,23}$ In intermediate- 2 and high-risk cases, it is worth it's worth trading off the adverse effects with desease symptoms, but this is not the case in the relatively quiescent risk categories of the disease.

The salutary effect of ruxolitinib and other Janus kinase inhibitors comes from their ability to inhibit JAK1 thus reducing the effects of pro-inflammatory cytokines. ${ }^{14}$ Therefore, the more JAK2-specific and JAK2 ${ }^{\mathrm{V} 617 \mathrm{~F}}$-specific inhibitors may not reduce the constitutional symptoms with the same intensity as that of ruxolitinib.

None of the Janus kinase inhibitors in development, including ruxolitinib, have a disease-modifying property in MPN nor do they lead to the eradication of the clone of MPN. ${ }^{44}$ None of these agents specifically targets the mutated JAK2 ${ }^{\mathrm{V} 617 \mathrm{~F}}$. There has been no clearance of mutated $J A K 2^{V 617 F}$ allele from the MPN patients with ruxolitinib. SAR302503 
did reduce the $J A K 2^{V 617 F}$ allele burden but there was no radical change. ${ }^{45}$ Though $J A K 2^{V 617 F}$ as a sole mutation can cause the MPN phenotype in mouse, in humans, whether it is the initiating mutation or not still awaits clarification; several MPN-specific mutations such as TET2 have often, but not always, been demonstrated to have appeared in MPN clone cells before the appearance of JAK2 $2^{V 17 F}$. Hence $J A K 2^{V 617 F}$ is not a founding mutation in MPN. Therefore, eradication of the disease-causing clones seems implausible with JAK2 inhibitors. ${ }^{46,47}$

There has been no reversal of fibrosis with any of the Janus kinase inhibitors, including ruxolitinib, in human disease within phase I/I or phase III trials, although ruxolitinib did yield histopathological responses in mouse models of $\mathrm{MF}^{16}$ For this reason, Janus kinase inhibitors cannot be looked upon as a panacea for MF.

The Mayo experience of extended follow up of phase II patients on ruxolitinib may not have been reproduced in phase III trials, but it is extremely interesting. There was a progressive escape from response to ruxolitinib over time. ${ }^{24}$ Ruxolitinib withdrawal syndrome was frequent and occasionally required hospitalization, ${ }^{48}$ although no such phenomenon has been ratified by the COMFORT studies. Loss of response and discontinuation of treatment was a common occurrence with ruxolitinib and other Janus kinase inhibitors in all the phase II trials of the other Janus kinase inhibitors across the board, which is quite alarming.

Spleen rate is only a soft end point for high-risk and intermediate-2 risk patients with MF. In the pre-statins era, a number of medications were introduced for hyperlipidemia, including nicotinic acid to reduce total and LDL cholesterol; only statins were seen to reduce mortality. Ruxolitinib performed well in terms of the soft end points and was shown to have a statistically significant survival benefit in a North American-Australian phase III trial (COMFORT I). ${ }^{22}$ The European trialists (COMFORT II) have failed to replicate this observation at least at the time of last publication of their results. ${ }^{23}$ Likewise, when the phase II data of ruxolitinib were presented by the two participating institutions, the MD Anderson experience suggested, by way of a proxy comparison, a survival advantage with ruxolitini ${ }^{25}$ that was not reflected in the Mayo experience. ${ }^{24}$ It is, therefore, clear that we need robust data from a phase III study, with probably a higher power, to document definite survival benefit with ruxolitinib in the intermediate- 2 and high-risk patients.

The ruxolitinib resistant mutations have already been documented in wild-type and V617F-mutated JAK2 ${ }^{49} \mathrm{~A}$ suspicion that can be raised is that an otherwise low-grade MF with a median survival of 3-5 years, after treatment with Janus kinase inhibitors might be converted to a high-proliferation phenotype due to genotypic variation. This could make the disease even more unamenable to control even with conventional medications.

Finally, whereas the non-leukemogenicity of hydroxyurea is time tested and amply testified in literature, ${ }^{50-51}$ the precise rate of leukemogenesis in MF has yet to be studied with ruxolitinib by way of a longterm follow up of non-responders.

\section{Conclusions}

Inhibitors of one or the other type of Janus kinase are likely to be of use in hematologic malignancies and in benign disorders such as rheumatoid arthritis and other autoimmune diseases. In MPN, the success of ruxolitinib in phase III trials and that of many other small-molecule JAK2 inhibitors in phase II trials in terms of spleen and constitutional response has given rise to the hope that an imatinib-like phenomenon could be repeated in MPN in the time to come with more JAK2 ${ }^{\mathrm{V} 617 \mathrm{~F}}$-specific inhibitors. It remains to be seen what consistent impact these agents will have on survival of the high-risk and intermediate-2 risk patients with MF, whether resistant mutations will lead to the effect attrition with time, what natural history the non-responders will have, what effect these agents will have on evolution to leukemia, whether a rebound in resistant phenotype will offset the gains during sensitive stage, whether these agents will find a place in low-risk and intermediate-1 risk patients of MF, and whether any meaningful health outcomes and replacement of hydroxyurea and aspirin will be possible with these drugs in non-myelofibrosis PV and ET.

\section{References}

1. Cervantes F, Dupriez B, Pereira A, et al. New prognostic scoring system for primary myelofibrosis based on a study of the International Working Group for Myelofibrosis Research and Treatment. Blood 2009;113:2895-901.

2. Barbui T, Thiele J, Passamonti F, et al. Survival and disease progression in essential thrombocythemia are significantly influenced by accurate morphologic diagnosis: an international study. J Clin Oncol 2011;29:3179-84.

3. Tefferi A, Rumi E, Finazzi G, et al. Survival and prognosis among 1,263 patients with polycythemia vera: an international study. Blood (ASH Annual Meeting Abstracts) 2011;118:277.

4. Baxter EJ, Scott LM, Campbell PJ, et al. Acquired mutation of the tyrosine kinase JAK2 in human myeloproliferative disorders. Lancet 2005;365:1054-61.

5. James C, Ugo V, Le Couédic JP, et al. A unique clonal JAK2 mutation leading to constitutive signalling causes polycythaemia vera. Nature 2005;434:1144-8.

6. Kralovics R, Passamonti F, Buser AS, et al. A gain-of-function mutation of JAK2 in myeloproliferative disorders. N Engl J Med 2005;352:1779-90.

7. Levine RL, Wadleigh M, Cools J, et al. Activating mutation in the tyrosine kinase JAK2 in polycythemia vera, essential thrombocythemia, and myeloid metaplasia with myelofibrosis. Cancer Cell 2005;7:387-97.

8. Zhao R, Xing S, Li Z, et al. Identification of an acquired JAK2 mutation in polycythemia vera. J Biol Chem 2005;280:22788-92.

9. Cross NC. Genetic and epigenetic complexity in myeloproliferative neoplasms. Hematology Am Soc Hematol Educ Program 2011;2011: 208-14.

10. Gnanasambandan K, Magis A, Sayeski PP. The constitutive activation of Jak2-V617F is mediated by a pi stacking mechanism involving phenylalanines 595 and 617. Biochemistry 2010;49:9972-84.

11. Hookham MB, Elliott J, Suessmuth Y, et al. The myeloproliferative disorder-associated JAK2 ${ }^{\mathrm{V} 617 \mathrm{~F}}$ mutant escapes negative regulation by suppressor of cytokine signaling 3. Blood 2007;109:4924-9.

12. Vainchenker W, Delhommeau F, Constantinescu SN, Bernard OA. New mutations and pathogenesis of myeloproliferative neoplasms. Blood 2011;118:1723-35.

13. Tefferi A. Novel mutations and their functional and clinical relevance in myeloproliferative neoplasms: JAK2, MPL, TET2, ASXL1, CBL, IDH and IKZF1. Leukemia 2010;24:1128-38.

14. Quintás-Cardama A, Kantarjian H, Cortes J, Verstovsek S. Janus kinase inhibitors for the treatment of myeloproliferative neoplasias and beyond. Nat Rev Drug Discov 2011;10:127-40.

15. Tefferi A. JAK inhibitors for myeloproliferative neoplasms: clarifying facts from myths. Blood 2012;119:2721-30.

16. Quintas-Cardama A, Vaddi K, Liu P, et al. Preclinical characterization of the selective JAK1/2 inhibitor INCB018424: therapeutic implications for the treatment of myeloproliferative neoplasms. Blood 2010;115:3109-17. 
17. Verstovsek S, Kantarjian H, Mesa RA, et al. Safety and efficacy of a JAK1 \& JAK2 inhibitor, INCB018424, in myelofibrosis. N Engl J Med 2010;363:1117-27.

18. Stein BL, Crispino JD, Moliterno AR. Janus kinase inhibitors: an update on the progress and promise of targeted therapy in the myeloproliferative neoplasms. Curr Opin Oncol 2011;23:609-16.

19. Verstovsek S, Passamonti F, Rambaldi A, et al. A phase 2 study of INCB018424, an oral, selective JAK1/JAK2 inhibitor, in patients with advanced polycythemia vera and essential thrombocythemia refractory to hydroxyurea. Blood (ASH Annual Meeting Abstracts) 2009;114:311.

20. Barosi G, Birgegard G, Finazzi G, et al. Response criteria for essential thrombocythemia and polycythemia vera: results of a European LeukemiaNet consensus conference. Blood 2009;113:4829-33.

21. Tefferi A. How I treat myelofibrosis. Blood 2011;117:3494-504.

22. Verstovsek S, Mesa RA, Gotlib J, et al. A double-blind, placebo-controlled trial of ruxolitinib for myelofibrosis. N Engl $\mathrm{J}$ Med 2012;366:799-807.

23. Harrison C, Kiladjian JJ, Al-Ali HK, et al. JAK inhibition with ruxolitinib versus best available therapy for myelofibrosis. $\mathrm{N}$ Engl $\mathrm{J}$ Med 2012;366:787-98.

24. Tefferi A, Litzow MR, Pardanani A. Long-term outcome of treatment with ruxolitinib in myelofibrosis. N Engl J Med 2011;365:1455-7.

25. Verstovsek S, Estrov Z, Cortes JE, et al. The MD Anderson Cancer Center (MDACC) Experience with ruxolitinib, an oral JAK1 and JAK2 inhibitor, in myelofibrosis: long-term follow-up outcomes of 107 patients from a phase I/I study. Blood (ASH Annual Meeting Abstracts) 2011;118:3851.

26. Pardanani A, Gotlib JR, Jamieson C, et al. Safety and efficacy of TG101348, a selective JAK2 inhibitor, in myelofibrosis. J Clin Oncol 2011;29:789-96.

27. Pardanani A, Gotlib J, Jamieson C, et al. SAR302503: interim safety, efficacy and long-term impact on JAK2 V617F allele burden in a phase I/II study in patients with myelofibrosis. Blood Blood (ASH Annual Meeting Abstracts) 2011;118:3838.

28. Pardanani A, Gotlib J, Gupta V, et al. An expanded multicenter phase I/I Study of CYT387, a JAK- 1/2 inhibitor for the treatment of myelofibrosis. Blood (ASH Annual Meeting Abstracts) 2011;118: 3849.

29. Tefferi A, Barosi G, Mesa RA, et al. International Working Group (IWG) consensus criteria for treatment response in myelofibrosis with myeloid metaplasia, for the IWG for Myelofibrosis Research and Treatment (IWG-MRT). Blood 2006;108:1497-503.

30. Pardanani AD, Caramazza D, George G, et al. Safety and efficacy of CYT387, a JAK-1/2 inhibitor, for the treatment of myelofibrosis.J Clin Oncol 2011;29 (Suppl; abstr: 6514).

31. Santos FP, Kantarjian HM, Jain N, et al. Phase 2 study of CEP-701, an orally available JAK2 inhibitor, in patients with primary or postpolycythemia vera/essential thrombocythemia myelofibrosis. Blood 2010;115:1131-6.

32. Hexner E, Goldberg JD, Prchal JT, et al. A multicenter, open label phase I/I study of CEP701 (lestaurtinib) in adults with myelofibrosis; a report on phase I: A study of the Myeloproliferative Disorders Research Consortium (MPD-RC). Blood (ASH Annual Meeting Abstracts) 2009;114:754.

33. Moliterno AR, Hexner E, Roboz GJ, et al. An open-label study of CEP-701 in patients with JAK2 V617F-Positive PV and ET: update of 39 enrolled patients. Blood (ASH Annual Meeting Abstracts) 2009;114:753.

34. Verstovsek S, Odenike 0, Scott B, et al. Phase I dose-escalation trial of SB1518, a novel JAK2/FLT3 inhibitor, in acute and chronic myeloid diseases, including primary or post-essential thrombo- cythemia/ polycythemia vera myelofibrosis. Blood (ASH Annual Meeting Abstracts) 2009;114:3905.

35. Deeg HJ, Odenike 0, Scott BL, et al. Phase II study of SB1518, an orally available novel JAK2 inhibitor, in patients with myelofibrosis. J Clin Oncol 2011;29 (Suppl; abstr: 6515).

36. Komrokji R, Wadleigh M, Seymour J, et al. Results of a phase 2 study of pacritinib (SB1518), a novel oral JAK2 inhibitor, in patients with primary, post-polycythemia vera, and post-essential thrombocythemia myelofibrosis. Blood (ASH Annual Meeting Abstracts) 2011;118:282.

37. Verstovsek S, Mesa R, Kloeker Rhoades S, et al. Phase I study of the JAK2 V617F inhibitor, LY2784544, in patients with myelofibrosis (MF), polycythemia vera (PV), and essential thrombocythemia (ET). Blood (ASH Annual Meeting Abstracts) 2011;118:2814.

38. Shah NP, Olszynski P, Sokol L, et al. A phase I study of XL019, a selective JAK2 inhibitor, in patients with primary myelofibrosis, post-polycythemia vera, or post-essential thrombocythemia myelofibrosis. Blood (ASH Annual Meeting Abstracts) 2008;112:98.

39. Rambaldi A, Dellacasa CM, Finazzi G, et al. A pilot study of the histonedeacetylase inhibitor givinostat in patients with JAK2 ${ }^{\mathrm{V} 617 \mathrm{~F}}$ positive chronic myeloproliferative neoplasms. Br J Haematol 2010; 150:446-55.

40. Mascarenhas J, Mercado A, Rodriguez A, et al. Prolonged low dose therapy with a pan-deacetylase inhibtor, panobinostat (LBH589), in patients with myelofibrosis. Blood (ASH Annual Meeting Abstracts) 2011;118:794.

41. DeAngelo DJ, Tefferi A, Fiskus W, et al. A phase II trial of panobinostat, an orally available deacetylase inhibitor (DACi), in patients with primary myelofibrosis (PMF), post essential thrombocythemia (ET), and post polycythemia vera (PV) myelofibrosis. Blood (ASH Annual Meeting Abstracts) 2010;116:630.

42. Guglielmelli P, Barosi G, Rambaldi A, et al. Safety and efficacy of everolimus, a mTOR inhibitor, as single agent in a phase 1/2 study in patients with myelofibrosis. Blood 2011;118:2069-76.

43. Begna K, Pardanani A, Mesa RA, et al. Pomalidomide therapy for myelofibrosis: analysis of results from three consecutive clinical trials. Blood (ASH Annual Meeting Abstracts) 2011;118:1759.

44. Passamonti F, Maffioli M, Caramazza D, Cazzola M. Myeloproliferative neoplasms: from JAK2 mutations discovery to JAK2 inhibitor therapies. Oncotarget 2011;2:485-90.

45. Passamonti F, Maffioli M, Caramazza D. New generation smallmolecule inhibitors in myeloproliferative neoplasms. Curr Opin Hematol 2012;19:117-23.

46. Wernig G, Kharas MG, Okabe R, et al. Efficacy of TG101348, a selective JAK2 inhibitor, in treatment of a murine model of JAK2 ${ }^{\mathrm{V} 617 \mathrm{~F}}$ induced polycythemia vera. Cancer Cell 2008;13:311-20.

47. Swierczek SI, Yoon D, Bellanne'-Chantelot C, et al. Extent of hematopoietic involvement by TET2 mutations in JAK2 ${ }^{\mathrm{V} 617 \mathrm{~F}}$ polycythemia vera. Haematologica 2011;96:775-778.

48. Tefferi A, Pardanani A. Serious adverse events during ruxolitinib treatment discontinuation in patients with myelofibrosis. Mayo Clin Proc 2011;86:1188-91.

49. Hornakova T, Springuel L, Devreux J, et al. Oncogenic JAK1 and JAK2-activating mutations resistant to ATP-competitive inhibitors. Haematologica 2011;96:845-53.

50. Bjorkholm M, Derolf AR, Hultcrantz M, et al. Treatment-related risk factors for transformation to acute myeloid leukemia and myelodysplastic syndromes in myeloproliferative neoplasms. J Clin Oncol 2011;29:2410-5.

51. Finazzi G, Caruso V, Marchioli R, et al. Acute leukemia in polycythemia vera: an analysis of 1638 patients enrolled in a prospective observational study. Blood 2005;105:2664-70. 